\title{
The fluid balance observed postnatally on normal pregnancies, pregnancies with preeclampsia, and on pregnancies with oedema and proteinuria
}

\author{
Athina Diamanti ${ }^{1,2}$, Paraskevi Katsaounou ${ }^{2}$, Victoria G. Vivilaki ${ }^{1}$
}

\begin{abstract}
INTRODUCTION It is observed that delivery does not completely eliminate the risk of developing symptoms of preeclampsia, as well as its impending complications. The postpartum period is often associated with a high risk of developing preeclampsia, eclampsia and HELLP syndrome. Yet, there is neither an established standard, nor specific guidelines for peripartum fluid management. Our purpose is to study during the first four days of the postpartum period the differences in fluid balance on: normal pregnancies, pregnancies with preeclampsia and pregnancies with oedema and proteinuria.

METHODS The setting was the Gaia private maternity clinic in Athens, where 100 women in the postpartum period took part in the study and were requested to fill in a data collection-sheet, specially developed for the study. They were recruited between January 2014 and November 2014 and were divided into three groups. Group I consisted of all normal pregnancies, Group II those with oedema and proteinuria, and Group III those with preeclampsia. The demographic and clinical characteristics of the women and fetuses were initially recorded. Subsequently, daily records of fluid balance, as well as body weight, were kept for four days after labor. Descriptive statistics were used to analyze the demographic data of the sampled women. We compared the fluid balances among the three groups using t-test association and one-way Anova.

RESULTS The mean age of the women was $32.48 \pm 4.38$ years. Out of a total of 100 women, 59 of them had a normal pregnancy (Group I), 24 developed oedema and proteinuria (Group II) and 17 developed preeclampsia (Group III). All three groups had a positive fluid balance on the first day postpartum and only the first group had a negative cumulative fluid balance from the second day postpartum and onwards. The second group despite having started to discard a small volume of fluid, from the second day postpartum, the balance remained positive throughout the next two days. The third group however, had a negative fluid balance only during the fourth day after labor.

conclusions Our results indicate a difference in the kinetics of fluids in women with preeclampsia, compared to those that had a normal pregnancy and postpartum period. Consequently, there appears to be a need for more rational management and follow up on fluid balance throughout labor.
\end{abstract}

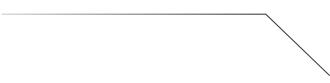 \\ AFFILIATION \\ 1 Department of Midwifery, Technological Educational Institute of Athens, Greece 2 Pulmonary Department, Intensive Care Medicine, Evaggelismos Hospital, Medical School, University of Athens, Greece}

\begin{abstract}
CORRESPONDENGE TO Athina Diamanti. Department of Midwifery, Technological Educational Institute of Athens, Greece, Tzermia 6, 15121 Pefki, Greece.

E mail: ath.diamanti@gmail.com
\end{abstract}

\section{KEYWORDS}

eclampsia, postpartum, preeclampsia, fluid balance, oedema, proteinuria

$\checkmark$

Received: 28 April 2017 Revised: 26 October 2017 Accepted: 1 December 2017

\section{INTRODUGTION}

Pregnancy involves remarkable changes in the anatomy and physiology that affect the renal and the cardiovascular system, in many aspects. These changes are in fact essential for the development and maintenance of a successful pregnancy, both for the mother and the fetus. Hemodynamic changes include an increase: in blood volume, in the maternal heart rate and in cardiac output (CO); but a decrease in arterial blood pressure and systemic vascular resistance. Mostly, all of these changes are generally reversed in weeks or months after delivery ${ }^{1-5}$.

To be more specific, pregnancy is a volume-expanding state characterized by net sodium retention of 900 to $1000 \mathrm{mEq}$, as well water retention of 1.1 to $1.6 \mathrm{~L}$, mediated by a balance between natriuretic and anti-natriuretic forces ${ }^{1}$. A rise in serum aldosterone is 
also observed, which results in a gain of approximately $1000 \mathrm{mg}$ of sodium ${ }^{6}$.

Tubular function and handling of water and electrolytes are altered, leading to mild increases in proteinuria, glucosuria, lower serum osmolality, and reduction in serum sodium levels. There is a net gain of sodium and potassium, but a greater retention of water, and a $30 \%$ to $50 \%$ increase in blood volume compared to non-pregnant women ${ }^{1}$. In normal pregnancy there is indeed an increase in total urinary protein and albumin excretion, specifically notable after 20 weeks. The rise in proteinuria during pregnancy is often attributed to the rise in the glomerular filtration rate (GFR). There is evidence that the amount of albuminuria increases in late pregnancy, yet with levels that do not exceed the upper limit of normal. Abnormal proteinuria in pregnant women is defined as protein levels of $300 \mathrm{mg}$ or more per 24 hours of urine collection, which is twice the normal limit in non-pregnant women. It can also be hinted by a dipstick measurement of ' $1+$ ' or more of protein per urine sample, based on NICE and ACOG ${ }^{1,7-9}$.

Proteinuria and decreased renal function are major indicators of preeclampsia. Both renal blood flow and glomerular filtration rate decrease in preeclamptic women compared to healthy pregnant women. Proteinuria develops in part secondary to impaired glomerular barrier integrity ${ }^{10}$.

Hypertensive disorders, on the other hand, are the most common medical complication of pregnancy, which affects $6 \%$ to $8 \%$ of all pregnancies. About $30 \%$ of hypertensive disorders are due to chronic hypertension, and $70 \%$ are due to gestational hypertension or preeclampsia9. The Morton (2015) research on primary aldosteronism, which is often underdiagnosed in pregnancy, showed that it is also nowadays one of the most common causes of secondary hypertension during pregnancy ${ }^{6}$.

Hypertension, defined as a systolic blood pressure of $\geq 140 \mathrm{mmHg}$ or diastolic blood pressure of $\geq 90 \mathrm{mmHg}$, is generally the earliest clinical indication of preeclampsia. It should be confirmed by at least two measurements. Abnormal proteinuria must also be present in order to make a confident diagnosis of preeclampsia. The level of hypertension can vary from mildly elevated blood pressure with minimal clinical significance, to severe hypertension and multiorgan dysfunction. These disorders are, unfortunately, responsible for significant matemal and perinatal morbidity and mortality 9 .

Preeclampsia occurs in $3-14 \%$ of pregnancies and is defined by maternal hypertension with abnormal proteinuria, generally also associated with oedema, coagulation abnormalities, and disseminated intravascular coagulation ${ }^{11}$. These conditions can also potentially lead to eclampsia, which is characterized by hyperreflexia and convulsions, and is associated with significant life-threatening complications like stroke, haemorrhage, oedema and brain herniation ${ }^{12}$.

Several other signs and symptoms are also associated with preeclampsia, such as oedema, rapid weight gain, headaches, dizziness, tinnitus, altered consciousness, epigastric or right upper quadrant pain, liver tenderness, and oliguria or anuria. Visual symptoms might also be present, as a result of retinal lesions ${ }^{7-9}$. In addition, pulmonary oedema, which is one of the more adverse potential complications of preeclampsia, has again an etiology that is multifactorial ${ }^{13}$.

Although most studies primarily focus on antenatal and intrapartum treatment, hypertension in the postpartum period is also a common phenomenon. There are few data regarding the incidence, risk factors, optimal treatment and outcome of hypertensive disorders that are diagnosed in the postpartum period ${ }^{14-16}$. It is recognized that the immediate postpartum period continues to be a period of risk in patients with preeclampsia, with up to $44 \%$ of the total eclamptic seizures occurring in this period; $44 \%$ of the cases of eclampsia developed antenatal, 33\% intrapartum and 23\% postpartum ${ }^{9,14-16}$; while $26 \%$ of seizures develop 0-48 hours after delivery ${ }^{14-16}$.

During the past decades, there has been extensive research regarding the incidence, risk factors, antepartum treatment, and outcome of women with preeclampsia; but there is little evidence on the management and the balance of fluids in the postpartum period. The proven reduction in serious complications of preeclampsia over the past few decades can be attributed to: prenatal care, the improved ability in detecting the signs and symptoms of preeclampsia earlier, and the prophylactic use of magnesium sulfate during labor and after delivery ${ }^{14-16}$.

Delivery, however, does not eliminate the risk for preeclampsia and its complications. Efforts should definitely be directed at continued monitoring, reporting and evaluating of preeclampsia symptoms during the postpartum period ${ }^{14-16}$. In such cases there are, unfortunately, no specific guidelines that would indicate the volume of fluids to be consumed orally, as well as the volume of intravenous fluid to be administered to peripartum women during the first few hours and first few days after delivery. In all, the peripartum fluid management approach generally remains controversial ${ }^{17-18}$.

The first 48 postnatal hours are very critical in terms of presenting pulmonary oedema symptoms, late postpartum preeclampsia or even eclampsia, and considering the physiological changes in the renal and the cardiovascular system that pregnant women have undergone that need time to be reverted, it is of paramount importance to establish a means of effective fluid balance monitoring and management, especially for women with preeclampsia, oedema and proteinuria.

These observations have been made despite the surprisingly limited information regarding normal urine output even in healthy women - as the guidance on monitoring normally refers just to the accepted minimum urine output of $40 \mathrm{~mL} / \mathrm{h}$ of the non-gravid state ${ }^{17}$.

The aim of the present study was to observe the difference in the balance of fluids among preeclamptic women, women with oedema and proteinuria, and those with a normal pregnancy, in the first four days postpartum.

\section{METHODS}

\section{Setting}

The study was conducted from January to November of 2014. The sample initially consisted of 130 women who gave birth in the maternity clinic Gaia in Athens, Greece. The selection was random and included both women who had vaginal birth, and women who delivered via a caesarian section.

\section{Design}

We compared three groups based on their fluid balance difference and their weight gain difference during 4 days postpartum. Unfortunately $23 \%$ of the sample ( 30 women) had to be eliminated from the study at a later time, either due to negligence from their side in keeping an accurate record or because they eventually became frustrated.

A data collection sheet was especially developed for the study in order to collect sociodemographic data such as age, gravida, kind of birth, gestation age, maternal weight, fetus weight and blood pressure. Following that, a daily record of the fluid balance and body weight was kept for four days after labor (Table 1). 
Research paper

\section{Table 1. Data collection sheet}

\begin{tabular}{|l|l|l|l|l|l|l|l|}
\hline Date & $\begin{array}{c}\text { Intravenous } \\
\text { fluids }\end{array}$ & $\begin{array}{c}\text { Oralfluids } \\
\text { intake }\end{array}$ & Dailyurine & Totalfluidintake & $\begin{array}{c}\text { Totalfluid } \\
\text { outtake }\end{array}$ & $\begin{array}{c}\text { Fluid } \\
\text { balance }\end{array}$ & $\begin{array}{c}\text { Body } \\
\text { weight }\end{array}$ \\
\hline $\begin{array}{l}\text { 1st day } \\
\text { postpartum }\end{array}$ & & & & & & \\
\hline $\begin{array}{l}\text { 2nd day } \\
\text { postpartum }\end{array}$ & & & & & & \\
\hline $\begin{array}{l}\text { 3rd day } \\
\text { postpartum }\end{array}$ & & & & & & \\
\hline $\begin{array}{l}\text { 4th day } \\
\text { postpartum }\end{array}$ & & & & & & \\
\hline
\end{tabular}

\section{Groups}

The sample was divided into 3 groups, based on whether or not they had developed oedema, proteinuria and hypertension, according to NICE and ACOG 2011 guidelines ${ }^{7-8}$ about the diagnosis and management of preeclampsia.

\section{Group I}

The first group comprised women who had a normal pregnancy with no hypertension or proteinuria observed.

\section{Group II}

The second group consisted of women who only had oedema (identified by clinical examination) and proteinuria (confirmed by chemical examination), but did not develop hypertension. Therefore, based on the guidelines, this group did not have preeclampsia.

\section{Group III}

The third group comprised women who had both hypertension (systolic blood pressure $>140 \mathrm{mmHg}$, diastolic blood pressure $>90 \mathrm{mmHg}$ ) and abnormal proteinuria (24-hour urine protein excretion greater than $300 \mathrm{mg})$.

\section{Data collection - procedures}

Initially, women were asked to give their consent in order to participate in the study. They were then extensively briefed on the purpose and

Table 2. Demographic and clinical characteristics of sample

\begin{tabular}{|c|c|c|c|c|}
\hline \multirow[t]{2}{*}{ Variates } & \multicolumn{3}{|c|}{ Mean $\pm S D$} & \multirow[t]{2}{*}{ Pvalue } \\
\hline & Group I & Group II & Group III & \\
\hline Age (years) & $\begin{array}{l}32.27 \\
\pm 3.59\end{array}$ & $\begin{array}{l}30.75 \\
\pm 4.24\end{array}$ & $35.65 \pm 5.57$ & $\begin{array}{c}0.003(I, I I) \\
0.10(I, I I)\end{array}$ \\
\hline $\begin{array}{l}\text { Blood pressure } \\
(\mathrm{mmHg}) \mathrm{SBP}^{*}\end{array}$ & $\begin{array}{l}107.3 \\
\pm 8.68\end{array}$ & $\begin{array}{l}120.8 \\
\pm 9.63\end{array}$ & $148.8 \pm 6.97$ & 0.0001 \\
\hline DBP** & $\begin{array}{l}64.66 \\
\pm 7.18\end{array}$ & $\begin{array}{l}75.42 \\
\pm 9.43\end{array}$ & $90.88 \pm 7.34$ & 0.0001 \\
\hline $\begin{array}{l}\text { Gestation age } \\
\text { (weeks) }\end{array}$ & $\begin{array}{l}38.33 \\
\pm 1.24\end{array}$ & $\begin{array}{r}40.40 \\
\pm 37.65\end{array}$ & $\begin{array}{c}35.34 \\
\pm 3.688\end{array}$ & $\begin{array}{c}0.0006(I,|I|) \\
0.86(I, I I)\end{array}$ \\
\hline $\begin{array}{l}\text { Maternal } \\
\text { weight gain } \\
\text { (kg) }\end{array}$ & $\begin{array}{l}12.36 \\
\pm 4.67\end{array}$ & $\begin{array}{l}14.54 \\
\pm 4.95\end{array}$ & $\begin{array}{c}13.59 \\
\pm 6.690\end{array}$ & 0.22 \\
\hline $\begin{array}{l}\text { Fetus weight } \\
\text { (kg) }\end{array}$ & $3.14 \pm 0.42$ & $2.88 \pm 0.93$ & $2.21 \pm 0.91$ & $0.0001(|| I I)$, \\
\hline
\end{tabular}

*Systolic Blood Pressure, **Diastolic Blood Pressure the aim of the study, and on the means of contribution expected of them. They were also reassured that there would be no disclosure of personal data. Consequently, they were given detailed instructions on the procedure of collecting the corresponding data and filling in the data collection sheet.

Participants were requested to keep a fluid balance diary (Table 1), and were instructed to record all oral input and urine output. The output was recorded under supervision by the midwife who would either empty the catheter bag or by using a measuring jug if the woman was not catheterized. In addition, a daily record of the participant's body weight was taken each morning by the staff.

The data collection sheet (Table 1), was specifically designed to meet the requirements of the study. During the procedure, women were continuously encouraged to discuss any concerns that they might have had. All participants were briefed verbally on the purpose of the study, and about preeclampsia symptoms and their effects after birth.

The fluid management scheme for all participants during labor, as well as during the first 4 postpartum days, was in accordance with the hospital protocols and anesthetist guidelines.

\section{Statistical and data analysis}

The statistical analysis was performed using the software package GraphPad Prism 5. Descriptive characteristics (including means, standard deviations, frequencies and percentages) were calculated for the sociodemographic variates. The assumptions of normality, homogeneity and independent cases of the sample were checked. T-test was carried out to compare the descriptive variables, as well as one-way ANOVA and Kruskal-Wallis tests to compare the descriptive variable of the observed fluid balance postnatally on normal pregnancies, on pregnancies with preeclampsia, and on pregnancies with oedema and proteinuria. Next, in order to further analyse the characteristics of the three groups, we compared the means, using the t-test and one-way ANOVA.

\section{Ethical considerations}

The research was conducted at the Gaia maternity clinic in Athens, Greece. The study protocol and the data collection sheet were both approved by the Ethical Committee of the Clinic, which granted the permission to conduct this study.

Oral consent was obtained from all the women, after being briefed on the type and purpose of the study. Each potential subject was adequately informed of the aims, methods and the anticipated benefits 
Table 3. Fluid balance

\begin{tabular}{|lcccc|}
$\begin{array}{l}\text { Fluidbalances } \\
(\mathbf{m L})\end{array}$ & \multicolumn{3}{c}{ Mean \pm SD } & Pvalue * $^{*}$ \\
& Group I & Group II & Group III & \\
\hline $\begin{array}{l}\text { Labour 1st day } \\
\text { postpartum }\end{array}$ & 1525 & 1585 & $1642 \pm 1610$ & 0.8946 \\
\hline 2nd day & \pm 1206 & \pm 1139 & & \\
postpartum & -21.53 & -55.83 & 178.1 & 0.6309 \\
\hline 3rd day & \pm 862.3 & \pm 1047 & \pm 1337 & \\
postpartum & -77.80 & 171.3 & 318.3 & 0.2963 \\
\hline 4th day & \pm 711.6 & \pm 1029 & \pm 1065 & \\
postpartum & -68.25 & 163.5 & -38.29 & 0.0144 \\
\hline
\end{tabular}

* Kruskal-Wallis test

Figure 1. Comparison of the mean age of the 3 groups

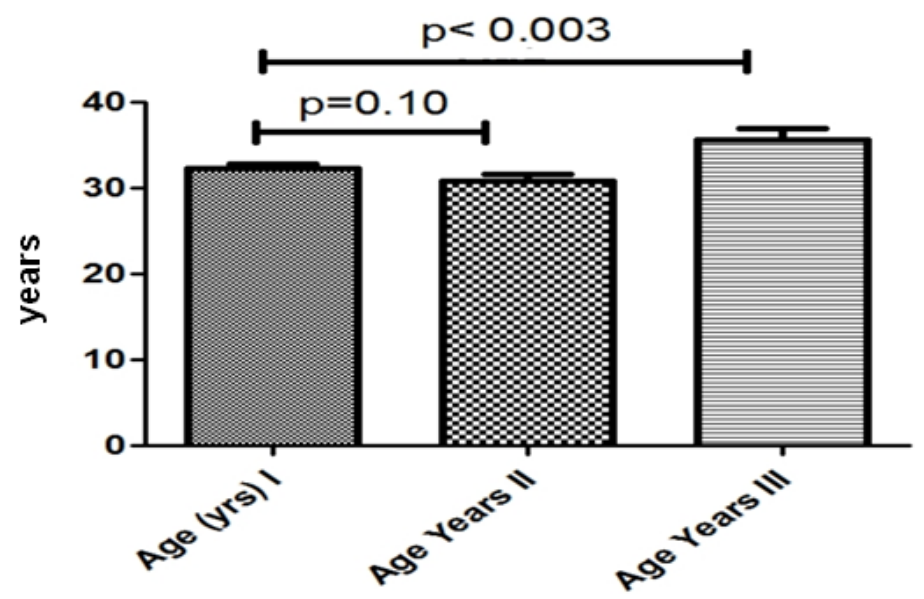

Figure 2. Comparison of the mean gestational age of the fetuses of the 3 groups

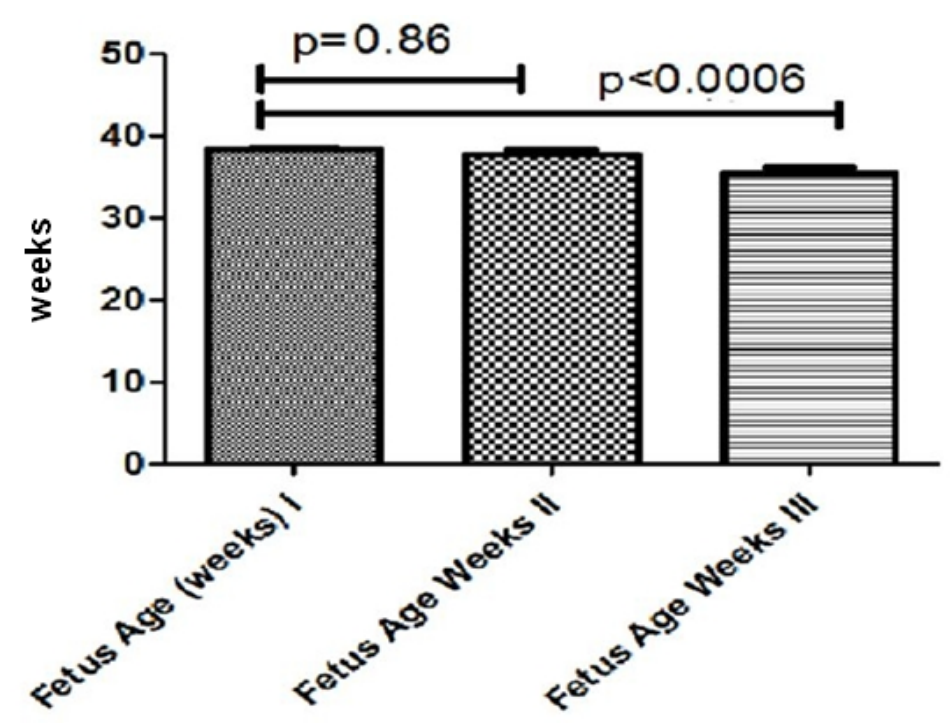

of the study. The women also reserved the right to refuse to participate in the study or to withdraw their participation at any time. Several precautionary measures were employed in order to protect the privacy of the research participants and the confidentiality of their personal information. The methods of the study were non-intrusive and were restricted only to the observation and recording of patient data, clearly stating that all answers would be confidential and that no personal data would be disclosed under any circumstances. No pervasive medical care actions were taken and no part of the standard care was omitted.

\section{RESULTS}

The data collected from 100 women were included in the final study analysis. The total participation rate was $77 \%$. The mean maternal age was $32.48 \pm 4.38$ years. The socio-demographic and clinical characteristics of the sample are shown in Tables 2 and 3.

The first analysis conducted was on the mean $( \pm S D)$ age difference of the three groups. As shown in Figure 1, the difference of the means of Group I and Group III, is actually statistically significant with a p-value $<0.003$, which clearly illustrates the considerable difference of age between the means of those two groups. On the other hand, the means of Groups I and II both with a p-value $=0.10$ demonstrate no statistical difference.

The second analysis conducted was on the mean $\left({ }^{ \pm} \mathrm{SD}\right)$ age difference of the fetuses of all the three groups during each pregnancy week. As shown in Figure 2, the difference on the means of Group I and that of Group III is statistically significant with a p-value $<0.0006$, which is indicatory of the considerable fetus age difference between the means of the two groups. Again the means $( \pm S D)$ of Groups I and II both with a $p$-value $=0.86$ demonstrate no statistical difference.

The third analysis conducted was on the means $( \pm S D)$ of the fetus birth weight. As the difference in the means $( \pm S D)$ of all three groups was assessed in parallel, a comparison using one-way Anova was selected. Consequently, as shown in Figure 3, a statistically significant difference was observed in the means of Group I and Group III, with a p-value < 0.0001, while between Groups I and II no such difference is observed. In addition, no significant difference is observed on the p-value of the 'weight gain' variate (the increase in a woman's weight throughout pregnancy), among the means of all three groups.

In addition, the comparisons conducted with the use of a KruskalWallis test among the three groups revealed a statistically significant difference only in the fluid balance of day 4 postpartum. Specifically, with regards to the fluid balance: on the 1st day postpartum $p=$ 0.8946, 2nd day postpartum $p=0.6309$, 3rd day postpartum $p=$ 0.2963 , and on the 4 th day postpartum $p<0.0144$ (Figure 4).

The mean $( \pm S D)$ Diastolic Blood Pleasure (DBP) and Systolic Blood Pleasure (SBP) values of the three groups of women were finally compared. An Anova comparison was selected, as the difference in the means $({ } \mathrm{SD})$ of all three groups was assessed in parallel. As shown in Figure 5, a statistically significant difference is observed on the DBP and SBP of all three groups, with a p-value $<0.0001$, for all individual comparisons performed.

\section{DISCUSSION}

This is probably the first ever attempt to investigate the fluid balance during the first four days of the postpartum period in a maternity setting. As mentioned previously, the observation of maternal 
Figure 3. Comparison of the weight gain of women and the weight of birth of the fetuses of the 3 groups

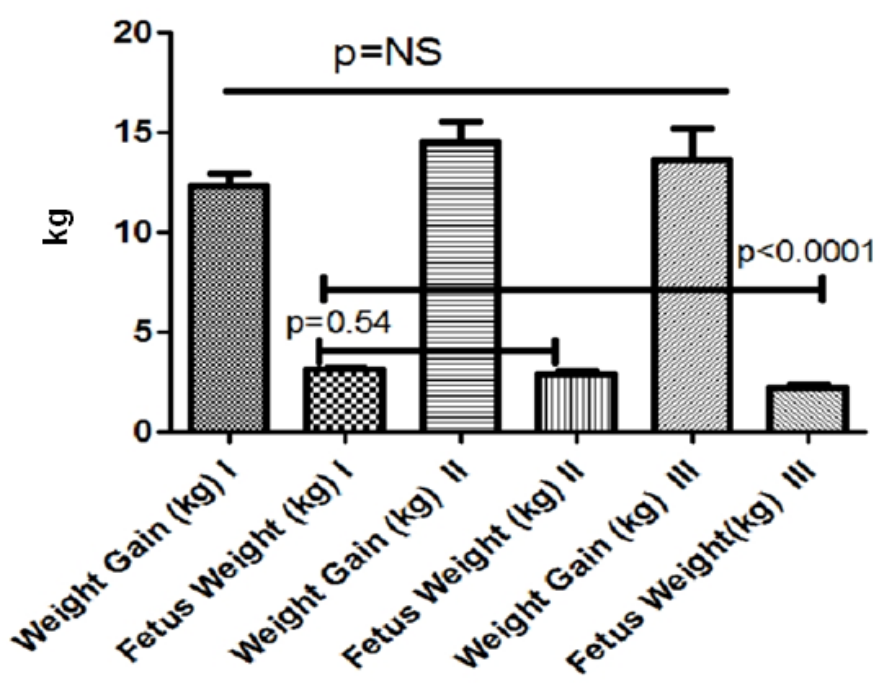
Figure 4. Comparison of the fluid balance over the first 4
days postpartum

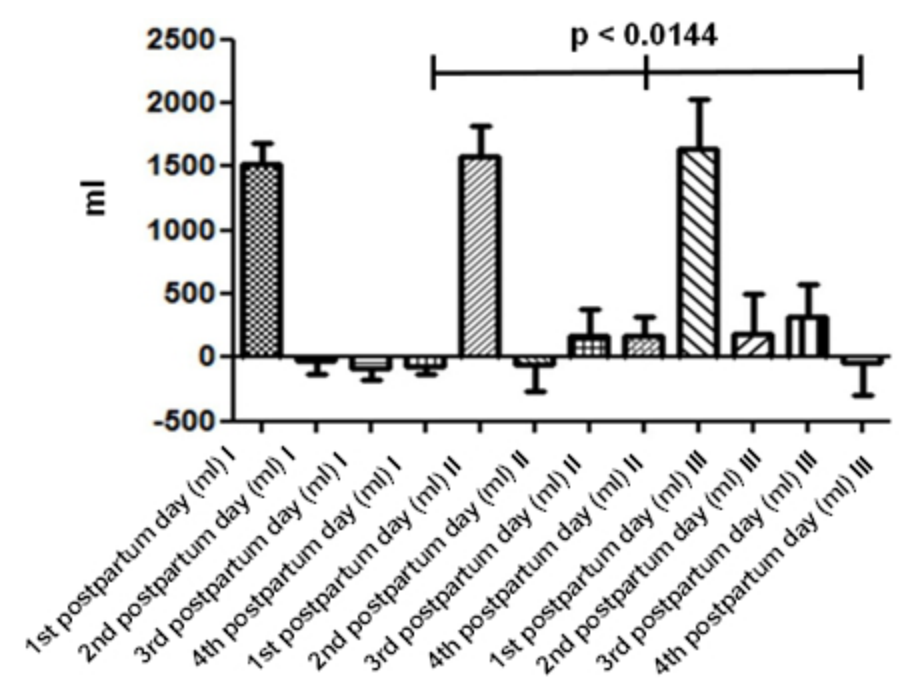

Figure 5. Comparison of the mean systolic and diastolic blood pressure of the 3 groups

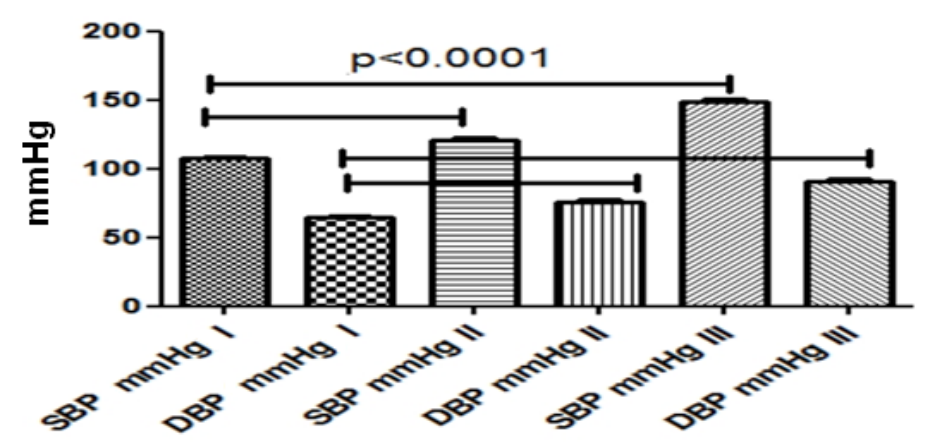

fluid balance during and after delivery is actually a challenge for the midwife, especially when a pregnancy is complicated by preeclampsia.

We observed that there were differences in the fluid balance among the three groups. More specifically all three groups had a positive fluid balance on the 1st day postpartum, and only the first group had a negative cumulative fluid balance from the 2 nd day postpartum and on. The second group despite having started to discard a small volume of fluid from the 2nd day postpartum, the balance remained positive throughout the next two days. The third group had a negative fluid balance only during the fourth day after labor. Statistical significant differences between groups were observed only on the fourth day postpartum.

Literature reports are conflicting and no large prospective outcome studies of fluid management have been performed ${ }^{19}$. A similar study to ours was conducted in a Chelsea hospital in Great Britain by Makenzie et al. ${ }^{17}$, which studied the fluid balance postnatally with emphasis, however, on the urine output rather than on the balance itself. Makenzie et al. studied a sample that involved 60 women who underwent caesarian section. The study duration however was 24 hours, compared to our 96 hours. It was found that the urine output varied widely between women, especially on the first 6 hours after delivery, where the median was $1 \mathrm{~mL} \mathrm{~kg}-1 \mathrm{~h}-1$. However, 6 to 12 hours after delivery, the median urine output was 2 $\mathrm{mL} \mathrm{kg}-1 \mathrm{~h}-1$, a value that was significantly greater than on the 6 th hour. It is worth noting that in women with preeclampsia the urine output was $0.5 \mathrm{~mL} \mathrm{kg-1} \mathrm{h-1} \mathrm{at} \mathrm{the} \mathrm{12th} \mathrm{hour,} \mathrm{possibly} \mathrm{due} \mathrm{to} \mathrm{renal}$ dysfunction, and as a consequence required closer attention. This finding is on par with our research as well, as our group of subjects with preeclampsia was also found to have positive fluid balance in the first 3 days postpartum, and required attention again from the fluid management perspective.

Another finding of the Makenzie et al. research was the observation that the group of women who received oxytocin infusion generally had a lower urine output rate in the duration of the infusion, because of the known antidiuretic effects of oxytocin ${ }^{17}$. To expand a little on this and to compare with our research, it must be noted that our sample in total (all three groups) was being infused with oxytocin during the first day postpartum, in adherence to the clinic's protocols and guidelines, to avoid postpartum hemorrhage.

Two more researches have studied the urine output after caesarean section in pregnant women. The first was conducted by Pouta et al..$^{20}$ in which women with normal pregnancy and women with preeclampsia were compared with non-pregnant women. The researchers found no particular difference in the urine output in the third trimester of pregnancy, compared to 1-3 days postpartum in normal pregnancy, despite an increase in atrial size and in the concentration of Atrial Natriuretic Peptide (ANP). However, a statistically significant postpartum increase in the levels of ANP, diuresis and natriuresis was noted for the subjects with preeclampsia.

The other study was conducted by Al-Takroni et al..$^{21}$ in a group of 207 women who had a caesarean section under general anesthesia. All subjects were healthy and had no signs of preeclampsia. The study examined the hourly urine output on the first 48 hours postpartum, in comparison with the oral fluid intake that was received. The urine output was found to be approximately $0.8 \mathrm{mLkg}-1 \mathrm{~h}-1$, thus quite similar to the results of Makenzie et al. ${ }^{17}$. 


\section{Research paper}

Another interesting field of discussion is the high risk in the development of preeclampsia in conjunction with pulmonary oedema in the postpartum period. Therefore, the probability of the appearance of pulmonary oedema and oliguria during the postpartum period must receive particular attention. In this direction, an early study that was performed in Aberdeen by Engelhard and MacLennan in 1999 actually indicated that $1 / 3$ of preeclamptic patients who developed interstitial pulmonary oedema, developed it up to 6 days after labor. The majority of the patients, however, actually developed it inside the first 48 to 72 hours postpartum, probably due to the mobilization of extravascular fluid. Having realized the danger of developing pulmonary oedema in the first 3 days postpartum, it is worth noting that - as observed in our study - the group that had preeclampsia during pregnancy also had a positive fluid balance during the 3 first days postpartum. Thankfully though, none of our subjects eventually developed pulmonary oedema during this period.

In our case, for all subjects that ended up having oliguria or anuria, diuretics were administered in order to maintain the renal system's good operation and to avoid any risk of them developing pulmonary oedema. These subjects had to eventually be excluded from our study however, since we had already had to adjust their fluid balance, by administering the diuretics. Nevertheless, it is worth noting that these patients' condition eventually had a favorable outcome, therefore the continuous fluid balance monitoring postpartum, definitely contributed towards this direction. Monitoring at least just the urine output on the 3 days postpartum would also have been detrimental.

Engelhard and MacLennan ${ }^{19}$ in their review about the fluid management in preeclampsia, stated that 'There is no evidence of long-term renal damage in preeclampsia, but there are strong suggestions that pulmonary oedema is linked to fluid administration. Problems arise with fluid balance if renal insufficiency and injudiciously large fluid loads coexist'. They reach two important conclusions:

1. 'The fluid management profile adopted in pregnancies with preeclampsia requires rigorous clinical assessment and continuous calculation of the fluid balance in order to avoid further complications'.

2. 'Most women will have a brief period of oliguria of up to 6 hours postpartum, which is quite common in the cases of preeclampsia even when there is no fluid restriction. Such condition does require awareness, as it can lead temporarily to compromised renal function. Yet, it seems that permanent renal damage is eventually not frequently reported'.

According to the results of our study this is very important in the postpartum period as well, as subjects tend to keep a positive fluid balance, thus the danger of complications remains.

\section{Limitations and suggestions for future research}

The fact that the hospital in which our study was conducted has an Intensive Care Unit (ICU), means that a high number of patients with pregnancy complications is generally received, compared to other clinics and maternity hospitals that do not have an ICU. Therefore, preeclamptic patients were more likely to be recruited as subjects for our study. This by itself is a limitation, but despite this the sample was random in all other aspects.

The fact that a positive fluid balance was recorded in all three groups on the day of the delivery remains puzzling. This could have been due to the intravenous administration of oxytocin - a substance known for its antidiuretic properties - to all subjects in adherence to the clinic's protocols, to protect them against postpartum hemorrhage, as discussed by Joo et al. ${ }^{22}$. This accounts for an additional limitation in our study. Breast feeding also increases the levels of oxytocin in the body, yet studying this was not part of our research. Nevertheless, its effects are actually worth studying, in future research.

The fluid preload of the woman, which is a normal practice in the case of epidural anesthesia, is also a factor that could have led to the positive fluid balance, as it could have contributed to fluid accumulation and pulmonary oedema, especially in the event of decreased renal function. In our sample all women underwent epidural analgesia and were overloaded with fluids. We would like to encourage further research to address some of these limitations.

Further, based on the Bungum et al. ${ }^{23}$ research findings, it was shown that on the 5th day postpartum a considerable change was observed, both in the Colloid Osmotic Pressure (COP), and in the movement of fluids. We would therefore like to suggest that in future studies the period of monitoring fluid balance should be expanded to at least 5 days and that results, both from healthy subjects, and from subjects with preeclampsia should be incorporated and analyzed something which was not done in the Bungum et al. work.

Moreover, oxytocin induction, breastfeeding and epidural analgesia are factors that should be taken into serious consideration in future research, when dealing with preeclampsia and the monitoring of fluid balance.

\section{CONGLUSION}

The results of the study indicate a difference in the kinetics of fluids in women with antenatal preeclampsia and demonstrate the subsequent substantial need for more careful and justified administration of fluids intrapartum. The importance of continuing to monitor the fluid balance postpartum is also demonstrated.

Delivery does not eventually eliminate the risk for preeclampsia and its complications. Therefore, the postpartum period continues to be a high-risk period for the development of preeclampsia. Consequently, all clinicians should be cautious in order to recognize the relevant symptoms in time.

\section{REFERENCES}

1 CheungK.L. Lafayette R.A. Renal Physiology of Pregnancy. Advances in Chronic Kidney Disease. 2013;20(3): 209-14. doi: 10.1053/j.ackd.2013.01.012.

2 Hunter S., RobsonS.C. Adaptation of the maternal heart in pregnancy. BrHeart J 1992;68, 540-543.

doi: 10.1136/hrt.68.12.540

3 Horowitz K.M., IngardiaC.J., BogidaA.F. Anemia in pregnancy. Clinics in Laboratory Medicine 2013;33, 281-291.

doi: 10.1016/j.cll.2013.03.016.

4 Adamova Z., Ozkan S., Khalil R.A. Vascular and Cellular Calcium in Normal and Hypertensive Pregnancy. Current Clinical Pharmacology 2009;4, 172-190. doi:10.2174/157488409789375320.

5 Bridges E.J., WombleS., WallaceM., McCartneyJ. Hemodynamic Monitoring in High-Risk Obstetrics Patients I.Critical Care Nurse 2003; 23, 53-62.

6 Morton A. Primary aldosteronism and pregnancy. Pregnancy hypertension. Received: August 1, 2015; Accepted: August 11, 2015; Published Online: August 12, 2015.

doi: 10.1016/j.preghy.2015.08.003.

7 National Institute for health and Care Excellence (NICE), Hypertension 


\section{Research paper}

in pregnancy: The management of hypertensive disorders during pregnancy. NICE clinical guidelines, No. 107. RCOG Press; 2010 Aug.

8 The American College Of obstetricians and Gynecologists. The diagnosis and management of preeclampsia and eclampsia clinical practice guideline. Version 1.0 Guideline No. 3 Date of publication - September 2011 Revision date - September 2013.

9 Magee L.A., Pels A., Helewa M. , Rey E., von Dadelszen P. Diagnosis, evaluation, and management of the hypertensive disorders of pregnancy: executive summary. Canadian Hypertensive Disorders of Pregnancy Working Group. J Obstet Gynaecol Can. 2014 May;36(5):416-41. doi: 10.1016/s1701-2163(15)30588-0.

10 Moran P, Lindheimer MD, Davison JM. The renal response to preeclampsia. Semin Nephrol. 2004 Nov;24(6):588-95. doi: 10.1016/j.semnephrol.2004.07.008.

11 Turner J.A. Severe Preeclampsia: Anesthetic Implications of the Disease and Its Management. American Journal of Therapeutics 2009;16, 284-288. doi: 10.1097/MJT.0b013e31816f6fd8.

12 Cipolla M.J., Kraig R.P. Seisures in women with preeclampsia: Mechanisms and management. Fetal and Maternal Medicine Review 2011;22, 91-108. doi: 10.1017/S0965539511000040.

13 SibaiB.M., Mabie W.C. Hemodynamics of preeclampsia. Clinical Perinatology 1991;18, 727-747.

14 Matthys LA, Coppage KH, Lambers DS, Barton JR, Sibai BM. Delayed postpartum preeclampsia: an experience of 151 cases. Am J Obstet Gynecol. 2004 May;190(5):1464-6. doi: 10.1016/j.ajog.2004.02.037.

15 Chames M.C., Livingston J.C., Ivester T.S., Barton J.R., Sibai B.M. Late postpartum eclampsia: a preventable disease? Am J Obstet Gynecol. 2002 Jun;186(6):1174-7. doi: 10.1067/mob.2002.123824.

16 Brady W.J, DeBehnkeD.J., Carter C.T. Postpartum toxemia : hypertension, edema, proteinuria and unresponsiveness in an unknown female. Journal of Emergency Medicine 1995;13, 643648. doi: 10.1016/0736-4679(95)00070-q.

17 Mackenzie M.J., Woolnough M.J., Barrett N., Johnson M.R., YentisS.M. Normal urine output after elective caesarean section: an observational study. International Journal of Obstetric Anesthesia 2010;19, 379-383. doi: 10.1016/j.ijoa.2010.06.005.

18 Ickx B.E. Fluid and blood transfusion management in obstetrics. European Journal of Anaesthesiology 2010;27, 1031-1035. doi: 10.1097/EJA.0b013e32833c30e3.

19 EngelhardtT., MacLennan F.M. Fluid management in pre-eclampsia. International Journal of Obstetric Anesthesia 1999;8, 253-259. doi: 10.1016/s0959-289x(99)80106-x.

20 Pouta A.M., Räsänen J.P., Airaksinen K.E., Vuolteenaho O.J., Laatikainen T.J. Changes in maternal heart dimensions and plasma atrial natriuretic peptide levels in the early puerperium of normal and pre-eclamptic pregnancies. British Journal of Obstetrics and Gynaecology 1996;103, 988-992.

doi: 10.1111/j.1471-0528.1996.tb09548.x.

21 Al-Takroni A.M., Parvathi C.K., Mendis K.B., Hassan S., Qunaibi A.M. Early oral intake after caesarean section performed under general anaesthesia. Journal of Obstetrics and Gynaecology 1999;19, 34-37.

doi: 10.1080/01443619965921

22 Joo K.W., Jeon U.S., Kim G.H., Park J., Oh Y.K., Kim Y.S., Ahn C., Kim S., Kim S.Y., Lee J.S., Han J.S. Antidiuretic action of oxytocin is associated with increased urinary excretion of aquaporin-2. Nephrol Dial Transplant 2004;19, 2480-2486.

doi: 10.1093/ndt/gfh413.

23 Bungum L., Tollan A., Oian P. Antepartum to postpartum changes in transcapillary fluid balance. British Journal of Obstetrics and Gynaecology 1990;97, 838-842. doi: 10.1111/j.1471-0528.1990.tb02580.x.
CONFLICT OF INTERESTS The authors have completed and submitted the ICMJE Form for Disclosure of Potential Conflicts of Interest and none was reported.

\section{FUNDING}

There was no source of funding for this research

\section{PROVEnANGe ANd PeER} REVIEW

Not commissioned; Externally peer reviewed 